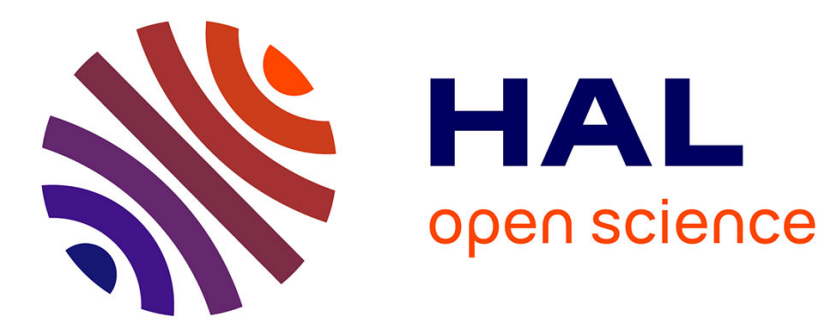

\title{
Nonepitaxially grown nanopatterned Co-Pt alloys with out-of-plane magnetic anisotropy
}

D. Makarov, F. Klimenta, S. Fischer, F. Liscio, S. Schulze, M. Hietschold, M. Maret, M. Albrecht

\section{- To cite this version:}

D. Makarov, F. Klimenta, S. Fischer, F. Liscio, S. Schulze, et al.. Nonepitaxially grown nanopatterned Co-Pt alloys with out-of-plane magnetic anisotropy. Journal of Applied Physics, 2009, 106, pp.114322. 10.1063/1.3260243 . hal-00954250

\section{HAL Id: hal-00954250 \\ https://hal.science/hal-00954250}

Submitted on 12 May 2020

HAL is a multi-disciplinary open access archive for the deposit and dissemination of scientific research documents, whether they are published or not. The documents may come from teaching and research institutions in France or abroad, or from public or private research centers.
L'archive ouverte pluridisciplinaire HAL, est destinée au dépôt et à la diffusion de documents scientifiques de niveau recherche, publiés ou non, émanant des établissements d'enseignement et de recherche français ou étrangers, des laboratoires publics ou privés. 


\title{
Nonepitaxially grown nanopatterned Co-Pt alloys with out-of-plane magnetic anisotropy
}

\author{
D. Makarovv, ${ }^{1, a)}$ F. Klimenta, ${ }_{1}^{1, b)}$ S. Fischer, ${ }^{2}$ F. Liscio, ${ }^{3, c)}$ S. Schulze, ${ }^{1}$ M. Hietschold, ${ }^{1}$ \\ M. Maret, ${ }^{3}$ and M. Albrecht ${ }^{1}$ \\ ${ }^{1}$ Institute of Physics, Chemnitz University of Technology, D-09107 Chemnitz, Germany \\ ${ }^{2}$ Department of Physics, University of Konstanz, D-78457 Konstanz, Germany \\ ${ }^{3}$ SIMAP, INP Grenoble-CNRS-UJF, 1130 Rue de la Piscine, BP75, 38402 Saint Martin d'Heres, France
}

A study on the structural and magnetic properties of 5-nm-thick Co-Pt alloy films grown on thermally oxidized $\mathrm{SiO}_{2} / \mathrm{Si}(100)$ substrates as well as on self-assemblies of spherical $\mathrm{SiO}_{2}$ particles with sizes down to $10 \mathrm{~nm}$ is presented. An out-of-plane easy axis of magnetization was stabilized at deposition temperatures as low as $250{ }^{\circ} \mathrm{C}$ in a broad composition range between 40 and 70 at. \%of Pt. Owing to the low deposition temperatures, no chemical long-range order is found. Thus, the strong outof-plane magnetic anisotropy is expected to be caused by anisotropic short-range order effects. The magnetic behavior of $\mathrm{CoPt}$ alloys with an equiatomic composition grown on arrays of $\mathrm{SiO}_{2}$ particles was found to be similar to those on planar substrates. Structural investigations using high-resolution transmission electron microscopy revealed that a continuous CoPt layer has been formed, covering the particle tops and connecting them. The magnetic CoPt caps exhibit an out-of-plane easy axis for all particle sizes; however, no pronounced difference in coercive field with particle size was observed, which is associated with the specific morphology of the film structure.

\section{INTRODUCTION}

For further increase in the storage density in magnetic hard disk drives, materials with high uniaxial out-of-plane magnetic anisotropy such as (001) textured FePt or CoPt alloys are required to provide sufficient thermal stability for the written bits of information. However, high annealing temperatures are required to form an $L 1_{0}$ chemical order in initially fcc-disordered alloys. ${ }^{1}$ Therefore, many studies are directed to lower the ordering temperature, for instance, by growing the alloys on appropriate buffer layers, such as $\mathrm{MgO}, \mathrm{Ag}, \mathrm{Cr}$, and $\mathrm{CrRu}^{2-5}$ or by utilizing surfactants to guide the formation of anisotropic local ordering during deposition. $^{6-8}$

Recently, a reduction in ordering temperature was reported for $\mathrm{CoPt}_{3}(111)$ thin films grown on a low energy surface of $\mathrm{WSe}_{2}(0001){ }^{9-11}$ In this case, a strong out-of-plane magnetic anisotropy is induced by a short-range anisotropic chemical order driven by Se segregation even for films grown at room temperature. ${ }^{8}$ Note that out-of-plane anisotropy direction is not expected neither in the disordered fcc structure nor in the $L 1_{2}$-ordered $\mathrm{CoPt}_{3}(111)$ phase. A similar behavior was also observed in thin $\mathrm{CoPt}_{3}(111)$ films grown on $\mathrm{Pt}(111)$ and $\mathrm{Ru}(0001)$ at elevated deposition temperatures of about $400{ }^{\circ} \mathrm{C}$ just before long-range $L 1_{2}$-type chemical ordering sets in. ${ }^{12,13}$ In addition, the presence of out-of-plane anisotropy was found in fcc-disordered CoPt films with an equiatomic composition grown on fused $\mathrm{SiO}_{2}$ substrates. ${ }^{14}$

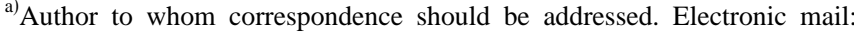
denys.makarov@physik.tu-chemnitz.de.

${ }^{b)}$ Present address: Max-Planck-Institut für Mikrostrukturphysik, D-06120 Halle, Germany.

${ }^{c)}$ Present address: CNR-IMM Sezione di Bologna, 40129 Bologna, Italy.
}

The physical origin of the short-range ordering resulting in the out-of-plane magnetic anisotropy is not yet clear. In this regard, Maranville et al. ${ }^{15}$ performed kinetic Monte Carlo (MC) simulations on continuous $\mathrm{CoPt}_{3}$ films relating the out-of-plane magnetic anisotropy to the short-range order induced by clustering of Co due to its step-edge segregation during the deposition process. This Co step-edge segregation does not depend on a particular growth direction. Therefore, the magnetic anisotropy orientation is expected to be independent of the growth direction, in agreement with experimental results reported by Shapiro et al. ${ }^{16}$ In contrast, MC simulations by Heinrichs et al. ${ }^{17,18}$ considered Pt segregation at the surface as a mechanism responsible for the short-range order effect in $\mathrm{CoPt}_{3}$ alloy nanostructures.

In this article, a detailed study on the magnetic and structural properties of 5-nm-thick $\mathrm{Co}-\mathrm{Pt}$ films grown on thermally oxidized $\mathrm{Si}(100)$ substrates is presented, including a careful investigation of the dependence on the growth temperature and composition of Co-Pt alloys. Furthermore, the combination of a template consisting of self-assembled nanoparticles with magnetic film deposition provides an elegant way of creating ordered arrays of nanostructures with welldefined magnetic properties. ${ }^{19-21}$ Therefore, it was investigated how these film properties will be altered by depositing 5-nm-thick CoPt alloys with an equiatomic composition onto self-assemblies of spherical $\mathrm{SiO}_{2}$ particles with various particle sizes down to $10 \mathrm{~nm}$.

\section{EXPERIMENTAL DETAILS}

The deposition of metal films was performed on planar thermally oxidized 100 -nm-thick $\mathrm{SiO}_{2} / \mathrm{Si}(100)$ substrates as well as on monolayers of self-assembled $\mathrm{SiO}_{2}$ particles. Par- 
ticle self-assemblies were prepared by using the convention routine proposed by Micheletto et al., ${ }^{22}$ where a droplet of a particle/water mixture is deposited onto a thermally oxidized silicon wafer. The samples were introduced into a molecular beam epitaxy chamber operating at a base pressure of 2 $\times 10^{-10}$ mbar. Metal deposition was carried out utilizing two electron beam sources. The composition of the codeposited Co-Pt alloy was controlled by a mass spectrometer adjusting the ratio between the individual Co and Pt fluxes. The deposition temperature, $T_{\mathrm{dep}}$, was varied between room temperature and $415{ }^{\circ} \mathrm{C}$. The total thickness of the Co-Pt alloys was chosen to be $5 \mathrm{~nm}$. An additional 1-nm-thick Pt capping layer was deposited at room temperature to protect the samples from oxidation.

The structural characterization has been carried out by $\mathrm{x}$-ray diffraction $(\mathrm{XRD})$ using a four-circle Huber diffractometer equipped with a $\mathrm{Cu} K \alpha$ rotating anode ( $\lambda$ $=1.5406 \AA$ ) and by scanning electron microscopy (SEM) and by high-resolution transmission electron microscopy (HRTEM) employing a $200 \mathrm{kV}$ Philips CM 20 FEG transmission electron microscope. The magnetic properties of the samples were investigated by a polar magneto-optical Kerr effect (P-MOKE) magnetometer using a focused laser beam with a wavelength of $670 \mathrm{~nm}$ and by a superconducting quantum interference device magnetometer in both in-plane and out-of-plane geometries. Magnetic force microscopy (MFM) was utilized to image the magnetic domain configurations of the samples at room temperature.

\section{RESULTS AND DISCUSSION}

\section{A. CoPt alloys grown on planar $\mathrm{SiO}_{2}$ substrates: Temperature dependence}

5-nm-thick CoPt alloy films with an equiatomic composition were grown on amorphous $\mathrm{SiO}_{2} / \mathrm{Si}(100)$ substrates at various growth temperatures between room temperature and $415^{\circ} \mathrm{C}$. The topography of the films was studied using atomic force microscopy (AFM), SEM, and transmission electron microscopy (TEM), revealing a grainy film morphology with a root mean square roughness of about $0.7 \mathrm{~nm}$ as well as a grain size of $(20 \pm 5) \mathrm{nm}$, which varies only slightly with deposition temperature up to $415^{\circ} \mathrm{C}$. An example is given in Fig. 1(a) showing a cross-sectional bright field TEM image performed on a film grown at $415{ }^{\circ} \mathrm{C}$.

In addition, XRD measurements reveal a [111] preferred orientation of grains along the surface normal (Fig. 2). The main structural properties deduced from the $\mathrm{x}$-ray patterns are summarized in Table I. The spacing of the fcc 111 planes is found to be very close to that in the bulk CoPt alloy $\left(d_{111}=2.18 \AA\right)$. The perpendicular coherence length, $L_{\text {perp }}$, deduced from the full width at half maximum (FWHM) of the 111 peaks increases with increasing deposition temperature, indicating an improvement of the (111) texture. The FWHM of the rocking curve measured at the 111 reflection for the sample grown at $415^{\circ} \mathrm{C}$ is equal to $10^{\circ}$ characterizing the poor degree of 111 grain orientation, in agreement with the HRTEM micrograph [Fig. 1(b)]. For the other samples grown at lower temperatures, the rocking curves are nearly flat, confirming the random orientation of 111 grains.
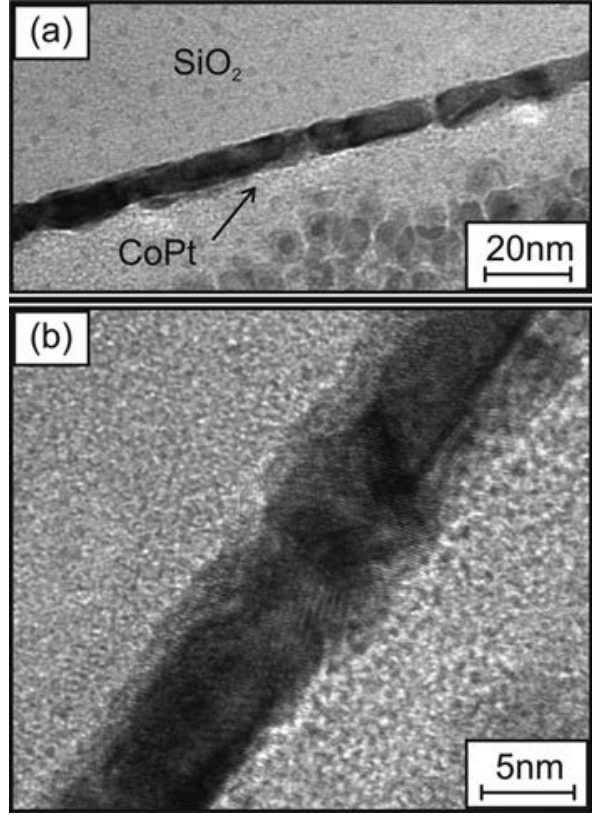

FIG. 1. (a) Cross-sectional bright field TEM study performed on a CoPt film grown at $415{ }^{\circ} \mathrm{C}$ revealing $\mathrm{CoPt}$ grains with a width of about $20 \mathrm{~nm}$. (b) HRTEM micrograph shows randomly oriented CoPt grains.

The magnetic characterization performed on the sample grown at RT reveals an in-plane magnetic anisotropy dominated by the shape anisotropy of the magnetic film. An anisotropy field of $H_{\mathrm{A}}=7.1 \mathrm{kOe}$ is extracted from the hard-axis loop (measurement in the out-of-plane geometry) [Fig. 3(a)]. Taking into account the saturation magnetization of the film $\left(M_{\mathrm{S}}=589 \mathrm{emu} / \mathrm{cm}^{3}\right)$, an effective anisotropy constant of $K_{\text {eff }}=H_{\mathrm{A}} M_{\mathrm{S}} / 2=-2.1 \times 10^{6} \mathrm{ergs} / \mathrm{cm}^{3}$ was estimated. The latter nearly matches the value of the shape anisotropy, $K_{\text {shape }}$ $=2 \pi M_{\mathrm{S}}^{2}=-2.2 \times 10^{6} \mathrm{ergs} / \mathrm{cm}^{3}$. Note that the saturation magnetization is found to be substantially smaller than the value reported for bulk CoPt alloys, which is in the range of $800 \mathrm{emu} / \mathrm{cm}^{3}{ }^{23-25}$ Although a lowering of the saturation magnetization is reported for nanostructured FePt films, ${ }^{26}$ the reason for the reduction of $M_{\mathrm{S}}$ in CoPt alloys grown on $\mathrm{SiO}_{2}$ substrates is not clear. The formation of dead magnetic layers at the interface between $\mathrm{CoPt}$ and $\mathrm{SiO}_{2}$ might be one of the possible explanations.

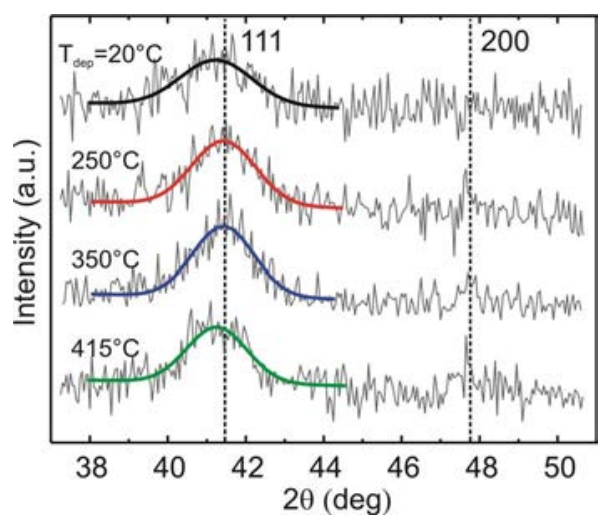

FIG. 2. (Color online) XRD $\theta$-2 $\theta$ diffraction patterns of 5-nm-thick CoPt alloy films grown on $\mathrm{SiO}_{2} / \mathrm{Si}(100)$ substrates at various temperatures. The positions of the fundamental 111 and 200 peaks for the bulk CoPt alloy are marked with dashed lines. 
TABLE I. Summary of the structural properties of 5-nm-thick CoPt alloy films grown on $\mathrm{SiO}_{2} / \mathrm{Si}(100)$ substrates at various temperatures. The XRD study was performed using $\mathrm{Cu} K \alpha$ radiation $(\lambda=1.5406 \AA)$. The position of the 111 peak, $2 \theta$, the FWHM of the peak, $\Delta(2 \theta)$, the perpendicular coherence length, $L_{\text {perp }}$, and the spacing of the fcc 111 planes, $d_{111}$, extracted from the peak position are listed.

\begin{tabular}{lcccc}
\hline \hline & $T_{\text {dep }}=20{ }^{\circ} \mathrm{C}$ & $250{ }^{\circ} \mathrm{C}$ & $350{ }^{\circ} \mathrm{C}$ & $415{ }^{\circ} \mathrm{C}$ \\
\hline $2 \theta$, deg & 41.24 & 41.43 & 41.42 & 41.24 \\
$\Delta(2 \theta)$, deg & 2.14 & 1.90 & 1.85 & 1.81 \\
$L_{\text {perp }}, \mathrm{nm}$ & 4.4 & 5.0 & 5.1 & 5.2 \\
$d_{111}, \AA$ & 2.19 & 2.18 & 2.18 & 2.19 \\
\hline \hline
\end{tabular}

The increase in the deposition temperature above $160{ }^{\circ} \mathrm{C}$ results in the appearance of a uniaxial magnetic anisotropy, leading to the stabilization of an out-of-plane easy axis of magnetization (Fig. 3). A maximum effective anisotropy value of about $2.6 \times 10^{6} \mathrm{ergs} / \mathrm{cm}^{3}$ was obtained for the sample deposited at $415{ }^{\circ} \mathrm{C}$ [Fig. 4(a)]. The increase in anisotropy is accompanied with an enhancement of the outof-plane coercive field, $H_{\mathrm{C}}$ [Fig. 4(b)]. In addition, an enhancement of the normalized out-of-plane remanence magnetization, $M_{\mathrm{R}} / M_{\mathrm{S}}$, was observed, as summarized in Fig. 4(c).

The presence of a strong out-of-plane magnetic anisotropy was also confirmed by analyzing the local magnetic domain configuration of the CoPt alloy in the demagnetized state using MFM imaging. A clear dark and bright contrast was observed, as shown in Fig. 5, where the increase in the deposition temperature from 250 to $415^{\circ} \mathrm{C}$ leads to a slight decrease in the average domain size from $(150 \pm 30)$ to $(120 \pm 30) \mathrm{nm}$.

Owing to the structural isotropy and the absence of the $L 1_{0}$ chemical order, the presence of neither out-of-plane easy axis of magnetization nor strong magnetic anisotropy is expected. The presence of an out-of-plane magnetic anisotropy in fcc $\mathrm{CoPt}_{3}$ films has already been reported ${ }^{13,14,27,28}$ and has been explained based on X-ray absorption fine structure measurements in terms of anisotropic chemical short-range order
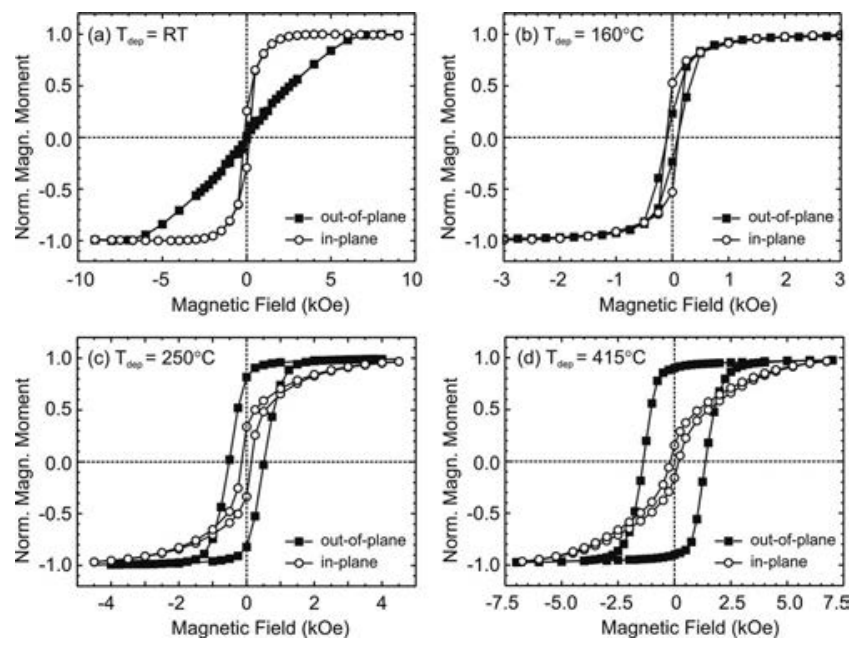

FIG. 3. Hysteresis loops measured in out-of-plane and in-plane geometries of the applied magnetic field for 5-nm-thick CoPt alloy films grown on $\mathrm{SiO}_{2} / \mathrm{Si}(100)$ substrates at various temperatures.

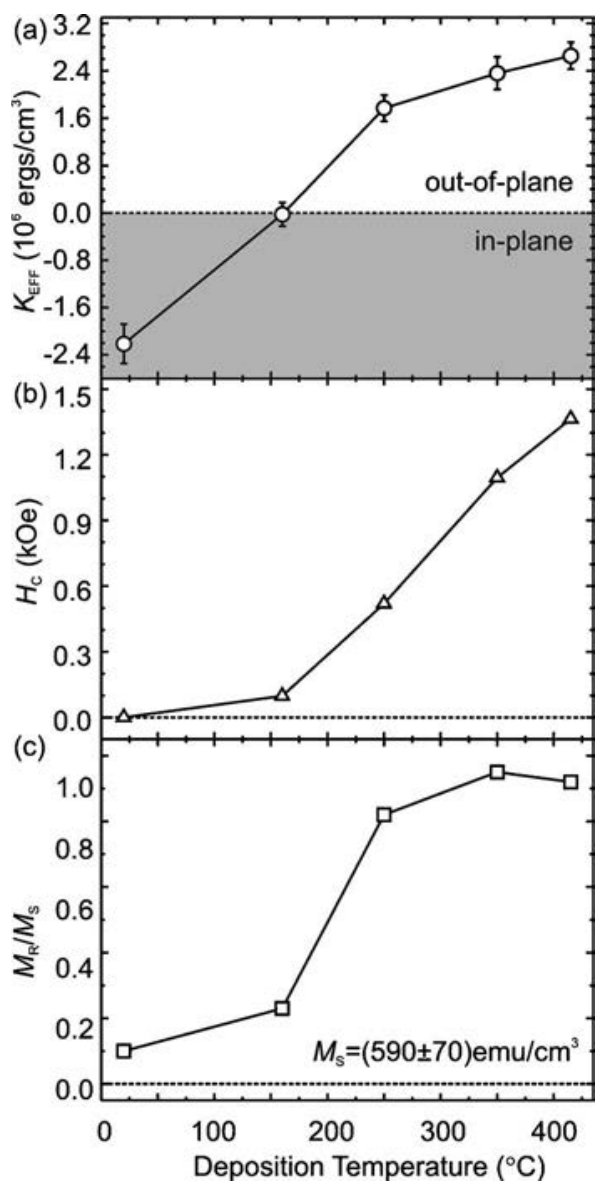

FIG. 4. The evolution of (a) the effective anisotropy constant, (b) out-ofplane coercive field, and (c) remanence magnetization with increasing deposition temperature.

due to the excess of $\mathrm{Co}-\mathrm{Co}$ nearest neighbors in the film plane driven by Pt segregation during codeposition. ${ }^{29}$ This effect leads to a strong anisotropy of the $3 \mathrm{~d}$ orbital moment of Co as well as to a weak anisotropy of the Pt orbital moment as deduced from angle-dependent X-ray magnetic circular dichroism measurements. ${ }^{28}$ However, other mechanisms, such as growth-induced heterogeneity ${ }^{16}$ or

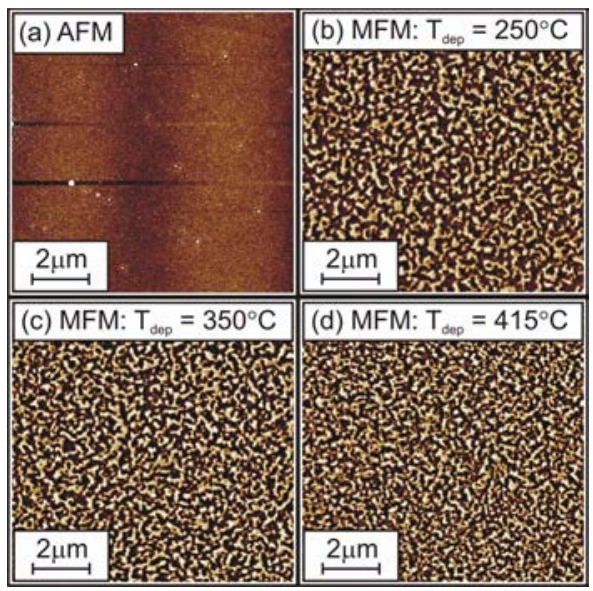

FIG. 5. (Color online) (a) AFM and [(b)-(d)] MFM images of 5-nm-thick CoPt films grown on $\mathrm{SiO}_{2} / \mathrm{Si}(100)$ substrates at various temperatures. The MFM images were taken at room temperature after demagnetizing the samples. 


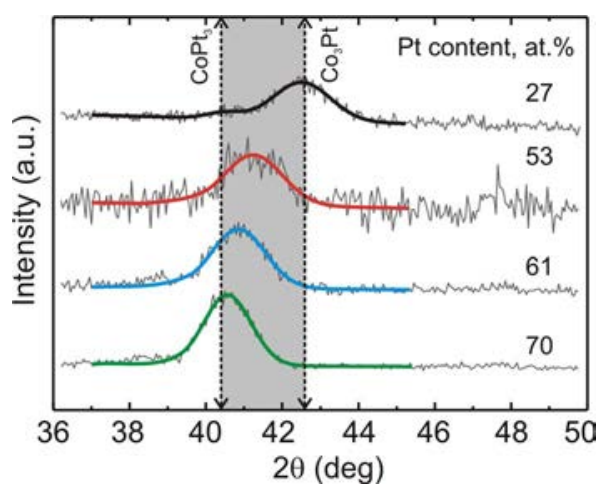

FIG. 6. (Color online) XRD $\theta-2 \theta$ diffraction patterns of 5-nm-thick Co-Pt films with various compositions. The positions of the 111 peak for the $\mathrm{CoPt}_{3}$ and $\mathrm{Co}_{3} \mathrm{Pt}$ alloys are marked with dashed lines. All samples were grown at $415{ }^{\circ} \mathrm{C}$ on amorphous $\mathrm{SiO}_{2} / \mathrm{Si}(100)$ substrates.

magnetically induced phase separation, ${ }^{12}$ have to be considered as well. Moreover, the composition modulation in $\mathrm{CoPt}$ thin films grown on $\mathrm{MgO}(111)$ single crystalline substrates might result in a formation of a chemically ordered $L 1_{1}$ phase with strong out-of-plane anisotropy ${ }^{30,31}$ In this case, the appearance of an additional superstructure peak at $2 \theta$ $\sim 21^{\circ}$ is expected. However, the texture quality of the investigated CoPt films is too poor to draw any conclusion supporting the possibility of the $L 1_{1}$ phase formation.

\section{B. Co-Pt alloys grown on planar $\mathrm{SiO}_{2}$ substrates: Composition dependence}

The stabilization of the out-of-plane easy axis is found to be strongly dependent on the composition of the Co-Pt alloys. ${ }^{14}$ Therefore, the structural and magnetic properties of Co-Pt alloy films with various compositions covering the range between $\mathrm{Co}_{0.25} \mathrm{Pt}_{0.75}\left(\mathrm{CoPt}_{3}\right)$ and $\mathrm{Co}_{0.75} \mathrm{Pt}_{0.25}\left(\mathrm{Co}_{3} \mathrm{Pt}\right)$ were investigated. For all these alloy films, the deposition temperature was chosen to be $415{ }^{\circ} \mathrm{C}$. XRD $\theta-2 \theta$ diffraction patterns are shown in Fig. 6. Similar to the XRD study presented above (Fig. 2), a (111) texture is observed. The position of the 111 peak shifts to higher $2 \theta$ values with decreasing $\mathrm{Pt}$ content, which corresponds to a reduction of the distance between 111 closest-packed planes. The extracted structural parameters from the XRD diffraction patterns are summarized in Table II.

The hysteresis loops of these samples are presented in Fig. 7. The sample with the lowest Pt content has an easy axis of magnetization in the film plane [Fig. 7(a)]. With in-

TABLE II. Summary of the structural properties of Co-Pt alloys with various $\mathrm{Pt}$ contents grown at $415{ }^{\circ} \mathrm{C}$. The XRD study was performed using $\mathrm{Cu} K \alpha$ radiation $(\lambda=1.5406 \AA)$. The position of the 111 peak, $2 \theta$, the FWHM of the peak, $\Delta(2 \theta)$, the perpendicular coherence length, $L_{\text {perp }}$, and the spacing of the fcc 111 planes, $d_{111}$, extracted from the peak position are listed.

27 at. $\%$ of Pt 53 at. $\%$ of Pt 61 at. $\%$ of Pt 70 at. $\%$ of Pt

\begin{tabular}{lcccc}
\hline $2 \theta$, deg & 42.47 & 41.24 & 40.85 & 40.56 \\
$\Delta(2 \theta)$, deg & 1.76 & 1.81 & 1.66 & 1.46 \\
$L_{\text {perp }}, \mathrm{nm}$ & 5.4 & 5.2 & 5.7 & 6.4 \\
$d_{111}, \AA$ & 2.12 & 2.18 & 2.21 & 2.22 \\
\hline
\end{tabular}
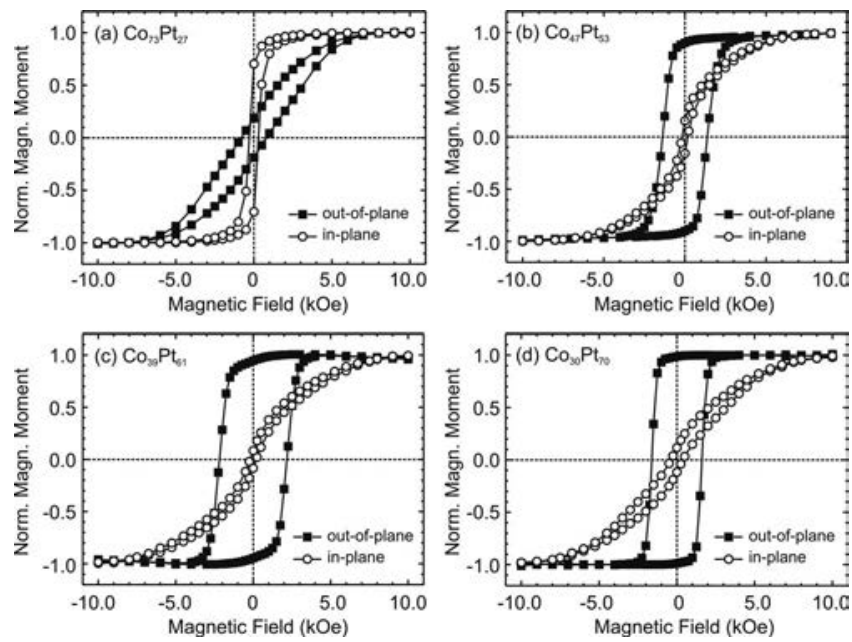

FIG. 7. Hysteresis loops measured in out-of-plane and in-plane geometries of the applied magnetic field for 5-nm-thick Co-Pt alloy films grown at $415{ }^{\circ} \mathrm{C}$.

creasing Pt content above 50 at. \%, a strong out-of-plane magnetic anisotropy was observed [Figs. 7(b)-7(d)]. The corresponding effective magnetic anisotropy values are plotted in Fig. 8(a). Note that in this composition range, the maximum value of effective anisotropy constant is found for the alloy with 53 at. \% of Pt. The increase of the Pt content in the alloy results in the gradual decrease of the $K_{\text {eff }}$ caused by the substantial reduction of the saturation magnetization [Fig. 8(b)].

\section{CoPt films grown on self-assembled arrays of $\mathrm{SiO}_{2}$ nanoparticles}

CoPt films with an equiatomic composition were deposited at $415{ }^{\circ} \mathrm{C}$ on self-assembled $\mathrm{SiO}_{2}$ particle arrays with
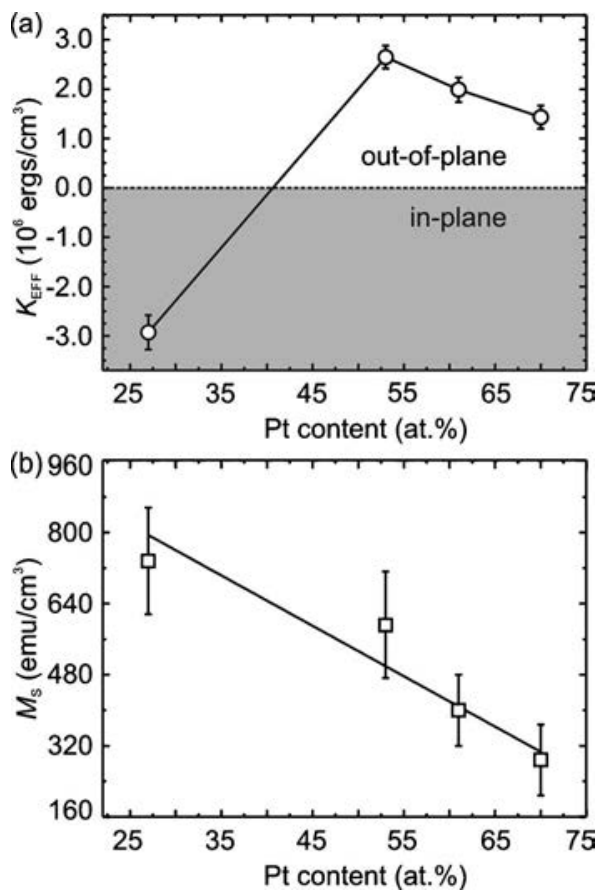

FIG. 8. The evolution of (a) the effective anisotropy constant and (b) the saturation magnetization with $\mathrm{Pt}$ content of 5-nm-thick Co-Pt alloy films grown at $415{ }^{\circ} \mathrm{C}$. 


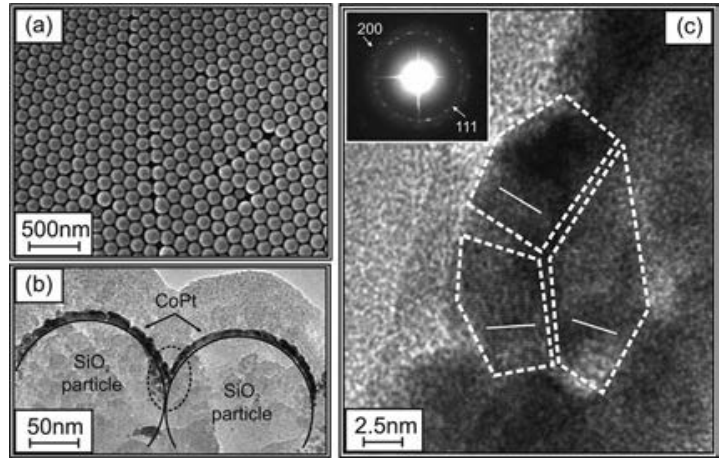

FIG. 9. CoPt alloy grown on an array of $\mathrm{SiO}_{2}$ particles with a diameter of $160 \mathrm{~nm}$. (a) Corresponding SEM image and (b) bright field cross-sectional TEM image. (c) The high-resolution TEM image revealing a polycrystalline structure of an individual CoPt grain. The white solid lines in (c) indicate the different orientations of the dense plane packing. The selected area electron diffraction pattern is presented as an inset in (c).

various particle diameters ranging from 10 to $900 \mathrm{~nm}$. An example of a densely packed array consisting of $160 \mathrm{~nm}$ particles is presented in Fig. 9(a). Note that owing to the particle size distribution, the pattern reveals some structural defects. The high-resolution TEM image in Fig. 9(c) shows that the cap consists of individual grains of typically $(8 \pm 5) \mathrm{nm}$ in lateral size with different orientations of the dense plane packing [marked with white lines in Fig. 9(c)]. The selected area electron diffraction image shown as an inset in Fig. 9(c) proves the polycrystalline nature of the CoPt film on the $\mathrm{SiO}_{2}$ particles. A bright field cross-sectional TEM image taken on this sample is shown in Figs. 9(b) and 9(c), revealing that neighboring film caps are clearly interconnected, resulting in direct magnetic exchange coupling in the magnetic cap array.

The magnetic properties of the films deposited on the particle arrays were investigated using P-MOKE magnetometry and MFM imaging. A series of P-MOKE hysteresis loops is shown in Fig. 10(a). The coercive field is found to be almost independent of the particle size. In this respect, the behavior of CoPt alloys differs substantially from the one observed on $\mathrm{Co} / \mathrm{Pd}(\mathrm{Pt})$ multilayers grown on arrays of polystyrene nanoparticles, where a scaling dependence of the coercive field on the particle size was found. ${ }^{20,21}$ This behavior is most likely due to the difference in film morphology, which gives rise to exchange coupling in the CoPt cap arrays. To get a better understanding of the magnetization reversal in the system, the angular dependence of the out-ofplane switching field was additionally investigated [Fig. 10(b)]. This has been done by measuring remanence curves using P-MOKE and by plotting the Kerr rotation signal at zero field after applying a reverse field under an angle to the initially saturated sample. The normalized switching field, $H_{\mathrm{S}} / H_{\mathrm{S}}\left(0^{\circ}\right)$, where $H_{\mathrm{S}}\left(0^{\circ}\right)$ is the switching field measured at $0^{\circ}$ (out-of-plane), is plotted in Fig. 10(b) versus the angle between the applied magnetic field and the direction normal to the sample plane. Surprisingly, the reversal behavior measured on particle arrays and on the planar substrate is found to be rather similar. However, the observed behavior differs from the one predicted according to the available theoretical models describing the magnetization reversal via a coherent
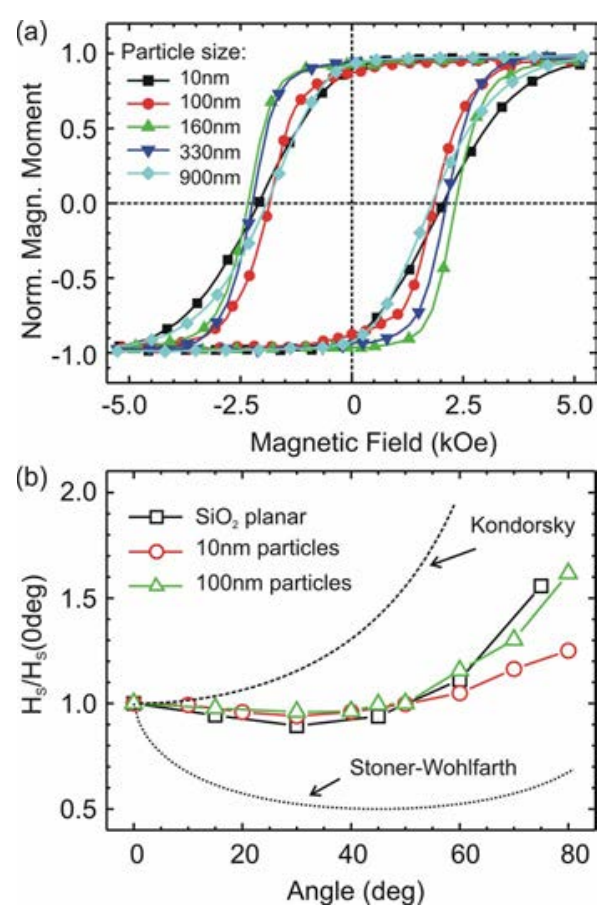

FIG. 10. (Color online) (a) MOKE hysteresis loops measured on the samples with various particle sizes. (b) Angular dependence of the switching field extracted from the remanence curves measured on the samples with 10 and $100 \mathrm{~nm}$ particles. The curve measured on the sample grown on a planar $\mathrm{SiO}_{2}$ substrate is also shown for comparison. The calculated curves for reversal mechanisms via domain wall motion and coherent rotation are also presented.

rotation of magnetization (Stoner-Wohlfarth model ${ }^{32}$ ) or via domain wall nucleation/propagation. ${ }^{33}$ A pronounced dispersion in magnetic easy axis orientation might be responsible for the weak angular dependence of the switching field [Fig. 10(b)]. In this case, the reversal is governed by many nucleation sites and limited domain wall propagation, as expected on the particle array. However, in order to make a final conclusion, a detailed micromagnetic modeling of the magnetization reversal is required.

MFM imaging is a powerful tool to locally visualize the magnetic domain configuration of the CoPt caps and to investigate the magnetic coupling in the particle arrays. A series of MFM images taken at remanence after acdemagnetizing the samples is presented in Fig. 11. Multidomain configurations are observed on $\mathrm{SiO}_{2}$ particles with sizes larger than $160 \mathrm{~nm}$ [Figs. 11(b)-11(d)], which is expected from the average domain size of about $120 \mathrm{~nm}$ measured on a CoPt film grown on a planar $\mathrm{SiO}_{2} / \mathrm{Si}(100)$ substrate [Fig. 11(a)]. With decreasing particle size down to $100 \mathrm{~nm}$, caps preferentially in a single-domain state are found [Fig. 11(e)]. This situation changes again for the deposit on the smallest particles of $10 \mathrm{~nm}$. In this case, the observed domain pattern appears to be rather similar to the one obtained on the planar $\mathrm{SiO}_{2} / \mathrm{Si}(100)$ substrate [compare Figs. 11(a) and 11(f)]. Therefore, it is suggested that a strong exchange coupling between neighboring caps is present and that magnetic domains can easily spread over the particle array.

In order to follow the magnetization reversal process in the magnetic cap arrays, MFM images had been taken at 


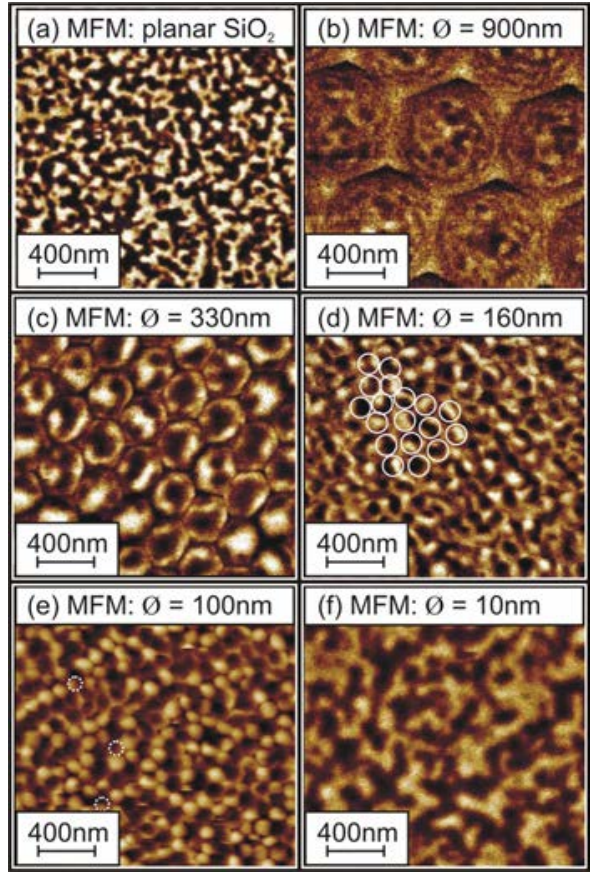

FIG. 11. (Color online) Series of MFM images taken at remanence on the samples with different particle sizes after ac demagnetizing: (b) $900 \mathrm{~nm}$, (c) $330 \mathrm{~nm}$, (d) $160 \mathrm{~nm}$, (e) $100 \mathrm{~nm}$, and (f) $10 \mathrm{~nm}$ particles. The position of the underlying particle template is marked for clarity. The dotted circles indicate several caps in a multidomain magnetic state. The domain pattern taken on a sample grown on a planar $\mathrm{SiO}_{2} / \mathrm{Si}(100)$ substrate is shown for comparison in (a).

remanence after exposing samples to different reverse magnetic fields $H_{\mathrm{R}}$. Note that an out-of-plane reverse field was applied to the initially saturated samples. A series of selected MFM images for the $100 \mathrm{~nm}$ particle array is shown in Fig. 12(a). In agreement with the P-MOKE hysteresis loop revealing a full remanence, the magnetization direction of the nanocaps after saturation remains unchanged at remanence. With the increase in the reverse field to $-1.5 \mathrm{kOe}$, several switching events, which are randomly distributed, occur. This shows the presence of various nucleation sites; however, at larger reverse fields, it appears that domain wall propagation sets in as well. The reversal of the $330 \mathrm{~nm}$ particle array shows the formation of multidomain states on the particle caps. From this comparison, in addition to the angle dependence of the switching field, it is suggested that rather exchange-coupled caps are present, leading to a reversal process that is dominated by nucleation which is widely spread accompanied by domain wall propagation across the particle arrays.

Furthermore, the particle template can also provide effective pinning sides for domain walls where effective domain wall pinning occurs when the magnetic domain wall width is comparable with the size of the pinning sites. ${ }^{34-37}$ In our films, the thickness of the Bloch domain wall is estimated to be about $18 \mathrm{~nm}$, which is of similar size compared to the areas where the caps are interconnected. A similar behavior was recently reported for CoPt alloys grown on arrays of $\mathrm{SiO}_{2}$ particles using 10-nm-thick $\mathrm{MgO}$ seed layers. ${ }^{38}$ However, an alternative explanation of the presence of single-domain caps in the exchange-coupled media could (a) Particles: $\emptyset=100 \mathrm{~nm}$ (b) Particles: $\varnothing=330 \mathrm{~nm}$

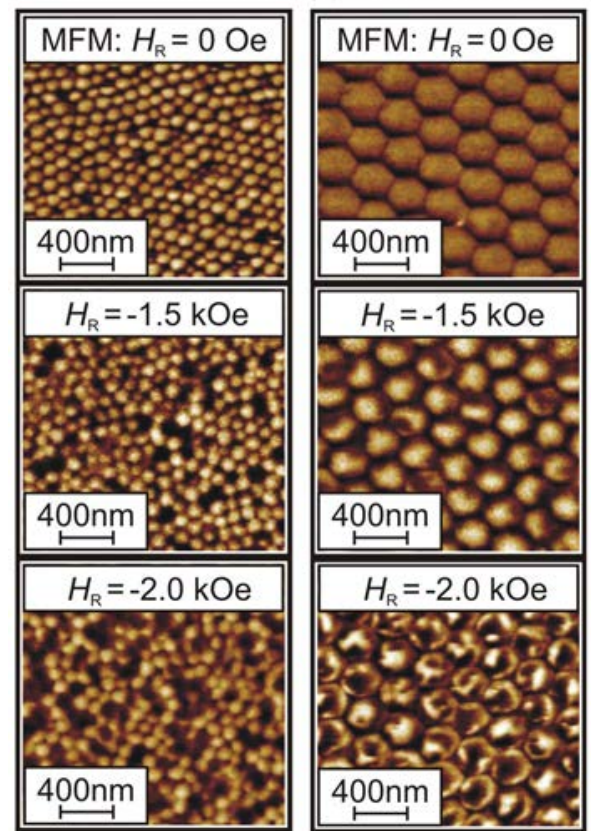

FIG. 12. (Color online) Series of MFM images taken on (a) $100 \mathrm{~nm}$ and (b) $330 \mathrm{~nm}$ particles at remanence after exposing them to a reverse magnetic field, $H_{\mathrm{R}}$, perpendicular to the substrate. Note that the images were taken at different locations on the sample.

be related to the formation of the bubble domains with a domain wall at the rim of a particle. Indeed, the magnetic parameters of the CoPt films used in the study fulfill the stability requirements for bubble domain formation. ${ }^{39-42}$

\section{CONCLUSIONS}

A study on the structural and magnetic properties of nonepitaxial Co-Pt alloy films grown on amorphous $\mathrm{SiO}_{2}$ substrates was presented. It turns out that hard magnetic $\mathrm{Co}-\mathrm{Pt}$ thin films with out-of-plane anisotropy can be formed at deposition temperatures as low as $250{ }^{\circ} \mathrm{C}$ in a broad composition range between 40 and 70 at. $\%$ of Pt. The structural origin of this out-of-plane magnetic anisotropy in textured films is attributed to the anisotropic short-range order effect; however, this needs to be further investigated.

A CoPt alloy with an equiatomic composition was then deposited on arrays of $\mathrm{SiO}_{2}$ particles of various sizes. The magnetic properties appear to be independent of particle size and similar to those found for films grown on planar substrates concerning $M_{\mathrm{R}} / M_{\mathrm{S}}$, coercive field $H_{\mathrm{C}}$, and angledependent switching. This behavior is related to the specific morphology of the cap structures where domain wall pinning at the rim of the cap might play an important role in the magnetization reversal process.

\section{ACKNOWLEDGMENTS}

The authors would like to thank B. Mainz (TU Chemnitz) for assistance in TEM measurements and A. Teichgräber (TU Chemnitz) for the preparation of $\mathrm{SiO}_{2}$ particle assemblies. The financial support by the European Commission through the project TERAMAGSTOR (Grant No. FP-7- 
224001) and by the Deutscher Akademischer Austauschdienst (Procope) is gratefully acknowledged.

${ }^{1}$ K. R. Coffey, M. A. Parker, and J. K. Howard, IEEE Trans. Magn. 31, 2737 (1995).

${ }^{2}$ Y. F. Ding, J. S. Chen, E. Liu, and L. Li, J. Magn. Magn. Mater. 303, e238 (2006).

${ }^{3}$ A.-C. Sun, J.-H. Hsu, H. L. Huang, and P. C. Kuo, J. Magn. Magn. Mater. 304, 106 (2006).

${ }^{4}$ Y. Xu, J. S. Chen, and J. P. Wang, Appl. Phys. Lett. 80, 3325 (2002).

${ }^{5}$ D. Makarov, C. Brombacher, F. Liscio, M. Maret, M. Parlinska, S. Meier, P. Kappenberger, and M. Albrecht, J. Appl. Phys. 103, 053903 (2008).

${ }^{6}$ B. B. Maranville and F. Hellman, Appl. Phys. Lett. 81, 4011 (2002).

${ }^{7}$ D. Makarov, F. Liscio, C. Brombacher, J. P. Simon, G. Schatz, M. Maret, and M. Albrecht, J. Magn. Magn. Mater. 320, 1858 (2008).

${ }^{8}$ M. Maret, B. Gilles, I. Guhr, B. Riedlinger, M. Albrecht, G. Schatz, and E. Beaurepaire, Nanotechnology 15, 1590 (2004).

${ }^{9}$ A. Maier, B. Riedlinger, F. Treubel, M. Maret, M. Albrecht, E. Beaurepaire, J.-M. Tonnerre, and G. Schatz, J. Magn. Magn. Mater. 240, 377 (2002).

${ }^{10}$ M. Albrecht, M. Maret, A. Maier, F. Treubel, B. Riedlinger, U. Mazur, G. Schatz, and S. Anders, J. Appl. Phys. 91, 8153 (2002).

${ }^{11}$ M. Maret, A. Maier, F. Treubel, B. Riedlinger, M. Albrecht, E. Beaurepaire, and G. Schatz, J. Magn. Magn. Mater. 242-245, Part 1, 420 (2002).

${ }^{12}$ P. W. Rooney, A. L. Shapiro, M. Q. Tran, and F. Hellman, Phys. Rev. Lett. 75, 1843 (1995).

${ }^{13}$ M. Maret, M. C. Cadeville, R. Poinsot, A. Herr, E. Beaurepaire, and C Monier, J. Magn. Magn. Mater. 166, 45 (1997).

${ }^{14}$ Y. Yamada, W. P. Van Drent, E. N. Abarra, and T. Suzuki, J. Appl. Phys 83, 6527 (1998).

${ }^{15}$ B. B. Maranville, M. Schuerman, and F. Hellman, Phys. Rev. B 73 104435 (2006).

${ }^{16}$ A. L. Shapiro, P. W. Rooney, M. Q. Tran, F. Hellman, K. M. Ring, K. L. Kavanagh, B. Rellinghaus, and D. Weller, Phys. Rev. B 60, 12826 (1999). ${ }^{17}$ S. Heinrichs, W. Dieterich, and P. Maass, Europhys. Lett. 75, 167 (2006).

${ }^{18}$ S. Heinrichs, W. Dieterich, and P. Maass, Phys. Rev. B 75, 085437 (2007).

${ }^{19}$ M. Albrecht, G. Hu, I. L. Guhr, T. C. Ulbrich, J. Boneberg, P. Leiderer, and G. Schatz, Nature Mater. 4, 203 (2005).

${ }^{20}$ T. C. Ulbrich, D. Makarov, G. Hu, I. L. Guhr, D. Suess, T. Schrefl, and M. Albrecht, Phys. Rev. Lett. 96, 077202 (2006).
${ }^{21}$ T. C. Ulbrich, D. Assmann, and M. Albrecht, J. Appl. Phys. 104, 084311 (2008).

${ }^{22}$ R. Micheletto, H. Fukuda, and M. Ohtsu, Langmuir 11, 3333 (1995).

${ }^{23}$ B. D. Cullity, Introduction to Magnetic Materials (Addison-Wesley, Reading, MA, 1972).

${ }^{24}$ M. R. Visokay and R. Sinclair, Appl. Phys. Lett. 66, 1692 (1995).

${ }^{25}$ S. H. Liou, Y. Liu, S. S. Malhotra, M. Yu, and D. J. Sellmyer, J. Appl. Phys. 79, 5060 (1996).

${ }^{26}$ C. Rong, D. Li, V. Nandwana, N. Poudyal, Y. Ding, Zh. Lin Wang, H. Zeng, and J. P. Liu, Adv. Mater. 18, 2984 (2006).

${ }^{27}$ T. A. Tyson, S. D. Conradson, R. F. C. Farrow, and B. A. Jones, Phys. Rev. B 54, R3702 (1996).

${ }^{28}$ W. Grange, M. Maret, J. P. Kappler, J. Vogel, A. Fontaine, F. Petroff, G. Krill, A. Rogalev, J. Goulon, M. Finazzi, and N. Brooks, Phys. Rev. B 58, 6298 (1998)

${ }^{29}$ C. Meneghini, M. Maret, V. Parasote, M. C. Cadeville, J. L. Hazemann, R. Cortes, and S. Colonna, Eur. Phys. J. B 7, 347 (1999).

${ }^{30}$ S. Iwata, S. Yamashita, and S. Tsunashima, IEEE Trans. Magn. 33, 3670 (1997).

${ }^{31}$ H. Sato, T. Shimatsu, Y. Okazaki, H. Muraoka, H. Aoi, S. Okamoto, and O. Kitakami, J. Appl. Phys. 103, 07E114 (2008).

${ }^{32}$ E. C. Stoner and E. P. Wohlfarth, Philos. Trans. R. Soc. London, Ser. A 240, 599 (1948).

${ }^{33}$ K. R. Coffey, T. Thomson, and J. U. Thiele, J. Appl. Phys. 92, 4553 (2002).

${ }^{34}$ Y. Tang and J.-G. Zhu, IEEE Trans. Magn. 43, 2139 (2007).

${ }^{35}$ Y. Tang and J. Zhu, IEEE Trans. Magn. 42, 2360 (2006).

${ }^{36}$ J. Zhu and Y. Tang, J. Appl. Phys. 99, 08 Q903 (2006).

${ }^{37}$ D. Suess, J. Fidler, K. Porath, T. Schrefl, and D. Weller, J. Appl. Phys. 99, 08G905 (2006).

${ }^{38}$ D. Makarov, E. Bermúdez-Ureña, O. G. Schmidt, F. Liscio, M. Maret, C. Brombacher, S. Schulze, M. Hietschold, and M. Albrecht, Appl. Phys. Lett. 93, 153112 (2008).

${ }^{39}$ A. A. Thiele, J. Appl. Phys. 41, 1139 (1970).

${ }^{40}$ J. E. Davies and E. A. Giess, J. Mater. Sci. 10, 2156 (1975).

${ }^{41}$ O. Hellwig, A. Berger, J. B. Kortright, and E. E. Fullerton, J. Magn. Magn. Mater. 319, 13 (2007).

${ }^{42}$ J. E. Davies, O. Hellwig, E. E. Fullerton, G. Denbeaux, J. B. Kortright, and K. Liu, Phys. Rev. B 70, 224434 (2004). 